\title{
Robustness of Water Resources Systems
}

\author{
TSUYOSHI HASHIMOTo ${ }^{1}$ \\ International Institute for Applied Systems Analysis, Laxenburg, Austria
}

Daniel P. Loucks and Jery R. Stedinger

School of Civil and Environmental Engineering, Cornell University, Ithaca, New York 14853

\begin{abstract}
When water resource systems investments are made there is little assurance that the predicted performance will coincide with the actual performance. Robustness is proposed as a measure of the likelihood that the actual cost of a proposed project will not exceed some fraction of the minimum possible cost of a system designed for the actual conditions that occur in the future. The robustness criterion is illustrated by its application to the planning of water supply systems in southwestern Sweden.
\end{abstract}

\section{INTRODUCTION}

Risk and uncertainty are characteristic of most planning situations. Water resource investment planning is no exception. Water resource projects often are large and expensive and require long lead times. Once the facilities are in place they are often operated for decades. Dams, pipelines, water and waste treatment facilities, canals, hydroelectric power plants, and water and sewer distribution networks are examples of such expensive long-lived investment projects. The uncertainty as to the level of service these facilities will need to provide in $5,10,20$, or 50 years from when they are planned and implemented makes the project evaluation and selection process difficult.

It is impossible to forecast the actual demand that a particular investment project will serve in the future. However, some project designs and operating policies may be sufficiently flexible to permit their adaptation to a wide range of possible demand conditions at little additional cost. Such systems can be called robust. This definition of robustness corresponds to Stigler's concept of economic flexibility [Stigler, 1939; Hashimoto, 1980b].

Others have used the term robustness in water resources planning to describe whether or not the optimal project design parameter values would remain essentially unchanged if the future demand conditions were to vary from those for which the project is designed [Fiering, 1976; Matalas and Fiering, 1977]. However, optimal design parameter values can be very sensitive to assumed future demand conditions, and this may not involve large economic opportunity costs [Loucks et al., 1981, pp. 122-129]. Thus it is appropriate to define system robustness in terms of the sensitivity of total system cost rather than the sensitivity of system design.

In this paper, robustness measures describe the overall economic performance of a water resource project. As such, they complement the more traditinal benefit cost and cost effectiveness criteria used for project selection. Other criteria designed to measure the dynamic system performance of

\footnotetext{
${ }^{1}$ Now with the International Development Center of Japan, Tokyo, Japan.

Copyright (C) 1982 by the American Geophysical Union.
}

projects are described in a companion paper [Hashimoto et al., this issue].

\section{MEASURES OF RoBUSTNESS}

Water resource project planning is based on forecasted or assumed future supplies, flows, qualities, costs, and benefits. It is also based on some assumed demand for the services the project is to provide. These assumed demand conditions, together with the environmental impacts and constraints that must be met, determine to a large extent the particular design, and hence the cost, of a project. In this paper all assumed future conditions that properly determine the actual motivation for and design of a project will be termed the 'demand conditions.'

Suppose a project is planned with a forecast of future demand conditions. If the forecast is not correct and another set of demand conditions actually occurs, the original project design may be inferior to another design better suited to the demand conditions that actually occurred. The difference between what the actual project costs and the costs that would be incurred with a cost effective design for the actual demand conditions is called the opportunity cost or regret. This is the cost of not having perfect information about the future.

Some projects may have the ability to adjust their final configuration or operating policies to the actual conditions as they evolve in the future, so that the opportunity cost of an original incorrect assumption about future demand conditions is reduced. Robustness measures should include the benefits and costs of such adjustments. If such modifications are cost effective for a reasonable range of future demand conditions, a project may be considered more desirable than one that is cost effective only for the most likely demand condition.

To define this concept more clearly, let $D$ denote a particular design and $q$ a future demand condition (e.g., wastewater flow, municipal water demand, low flow augmentation requirement, or level of flood protection desired). The function $C(q \mid D)$ will be the cost of accommodating the demand condition $q$ with the project design $D$. This cost includes the amortized construction, operation and maintenance costs, and the costs of any measures that need to be 


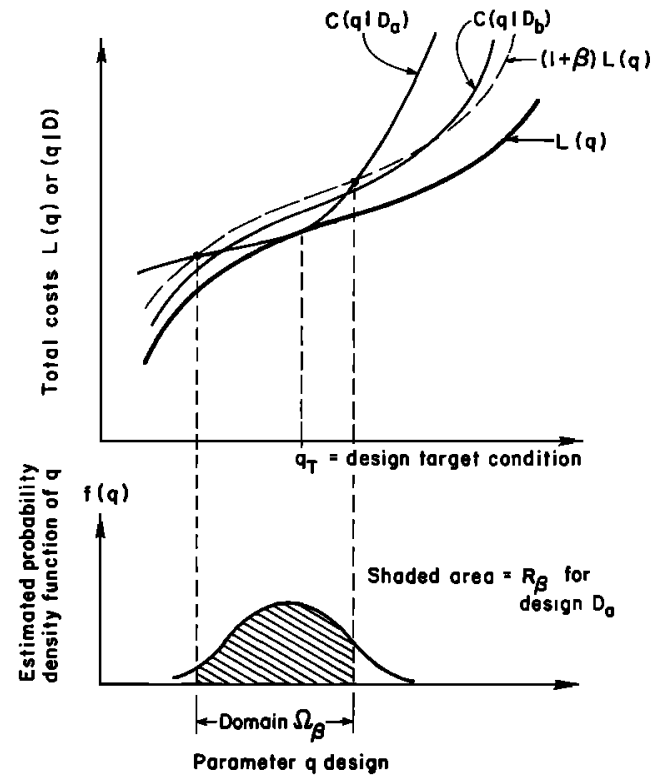

Fig. 1. For given $\beta$, robustness $R_{\beta}$ of design $D_{a}$ is the probability, given by shaded area in lower graph, that design $D_{a}$ 's cost $C(q)$ $\left.D_{a}\right)$ is not more than $(1+\beta) L(q)$.

taken to satisfy the actual demand conditions with design $D$.

Of interest for any demand condition $q$ is the minimum cost $L(q)$ of a design that can satisfy that assumed demand condition

$$
L(q)=\min _{\text {all } D} C(q \mid D)
$$

For any demand condition $q$ the opportunity cost of selecting design $D$ is the difference between the actual cost $C(q \mid D)$ and the minimum cost $L(q)$ of a design that satisfies $q$.

When examining the merits of a particular design $D$, one might ask for what values of demand conditions $q$ is the opportunity cost of $D$ no greater than a fraction $\beta$ of the minimum cost $L(q)$. If this set of $q$ values includes all those values of $q$ that could conceivably occur, then the cost of $D$ will always be within $100 \beta \%$ of the cost of the cost effective design no matter what the value of $q$. Thus attention is reasonably directed to those values of $q$ for which

$$
C(q \mid D) \leq(1+\beta) L(q)
$$

or

$$
\frac{C(q \mid D)-L(q)}{L(q)} \equiv R(q \mid D) \leq \beta
$$

for a given $\beta$ and design $D$.

The opportunity cost ratio $R(q \mid D)$ defined in (3) is the opportunity cost or regret divided by the minimum cost. This ratio is a measure of the relative magnitude of the opportunity cost of design $D$. This ratio may be more meaningful to some than the opportunity cost itself.

It is likely, especially for relatively small values of $\beta$, that no system design alternative $D$ will satisfy (2) or (3) for all conceivable future demand conditions $q$. This suggests that a probabilistic description of system robustness may be advantageous. Assume that one can assign probabilities to the likely future demand condition values of $q$. This defines the probability density function $f(q)$. Now possible system performance can be described, in part, by the expected opportunity cost.

$$
E_{q}[C(q \mid D)-L(q)]=\int_{-\infty}^{+\infty}[C(q \mid D)-L(q)] f(q) d q
$$

or by the expected utility of system cost,

$$
E_{q}[U(C(q \mid D))]=\int_{-\infty}^{+\infty} U(C(q \mid D)) f(q) d q
$$

[Friedman and Savage, 1948; Raiffa, 1968].

While utility theory provides an appropriate solution to the problem of design selection under risk or uncertainty, there are a number of reasons why its use has been limited in practice [Loucks et al., 1981]. Use of expected opportunity costs or, equivalently, the use of expected costs, is reasonable and commonly done. However, these expected costs provide little insight into how confident one can be that a particular design $D$ will be near or reasonably close to the least cost design. This need can be met by defining design robustness $R_{\beta}$ as the likelihood or probability that (2) or (3) will be satisfied:

$$
R_{\beta}=\operatorname{Prob}[C(q \mid D) \leq(1+\beta) L(q)]
$$

\begin{tabular}{|c|c|c|c|c|c|c|}
\hline & \multicolumn{4}{|c|}{ Costs $C_{i j}$ for Design $D_{j}$} & \multirow{2}{*}{$\begin{array}{c}\text { Probability } \\
\text { of } \\
\text { Condition, } \\
P_{l}\end{array}$} & \multirow{2}{*}{$\begin{array}{l}\text { Least } \\
\text { Cost, } \\
L_{t}\end{array}$} \\
\hline & $D_{1}$ & $D_{2}$ & $D_{3}$ & $D_{4}$ & & \\
\hline \multicolumn{7}{|l|}{$\begin{array}{l}\text { Future demand } \\
\text { condition }\end{array}$} \\
\hline$q_{1}$ & 60 & 90 & 110 & 75 & 0.10 & 60 \\
\hline$q_{2}$ & 55 & 30 & 35 & 50 & 0.20 & 30 \\
\hline$q_{3}$ & 50 & 30 & 20 & 35 & 0.50 & 20 \\
\hline$q_{4}$ & 55 & 35 & 35 & 25 & 0.20 & 25 \\
\hline Maximum cost* & 60 & 90 & 110 & 75 & & \\
\hline Expected cost $\dagger$ & 53 & 37 & 35 & 40 & & \\
\hline Variance of cost & 11 & 316 & 675 & 200 & & \\
\hline
\end{tabular}

Other measures of economic robustness have also been proposed [Hashimoto, 1980b].

The concept of robustness defined by (6) is illustrated in

TABLE 1. Cost of Each Design-Outcome Combination and Design Comparison Based Upon Cost

*Best design $D_{1}$.

†Best design $D_{3}$. 
Figure 1 for a situation where $q$ is a scalar quantity. Two alternative designs are considered, $D_{a}$ and $D_{b}$. The alternative design $D_{a}$ whose cost is represented by the cost function $C\left(q \mid D_{a}\right)$ is designed for a demand condition $q_{T}$. The design $D_{a}$ may also result in the minimum cost at other demand conditions. However, design $D_{a}$ incurs relatively large opportunity costs for demand conditions significantly different from $q_{T}$. An explicit consideration of robustness may result in the selection of an alternative design $D_{b}$ which is robust at level $\boldsymbol{\beta}$ for a wider range of demand conditions, even though design $D_{b}$ is not cost effective for any $q$.

The value of robustness $R_{\beta}$ at the level $\beta$ is simply the probability that the system's opportunity cost $C(q \mid D)$ $L(q)$ will not exceed $\beta$ times the minimum total cost $L(q)$. It is the probability that the design parameter $q$ will have a value within the domain $\Omega_{\beta}$ shown in Figure 1. In symbols,

$$
R_{\beta}=\int_{\Omega_{\beta}} f(q) d q
$$

\section{AN EXAMPLE}

The usefulness of robustness measures can be illustrated by an example. Suppose that there are four design alternatives, $D_{j}$ for $j=1, \cdots, 4$, which have total costs $C_{i j}$ for four possible future demand conditions $q_{i}$ as shown in Table 1 . Table 1 also gives the probabilities of each $q_{i}$ and the cost $L_{i}$ of the most cost effective alternative for each $q_{i}$. Alternative $D_{j}$ is cost effective for future demand conditions $q_{i}$ when $j=$ $i$. Table 1 also reports the maximum cost that may be incurred with each design, the expected cost, and the variance of costs. These criteria can be used for decisionmaking [Fabrycky and Thuesen, 1980]. One can insure that costs do not exceed 60 by choice of design $D_{1}$ which has the minimum maximum cost. The table also shows that design $D_{3}$ achieves the minimum expected cost. However, $D_{2}$ has only a slightly higher expected cost while the variance of costs is much lower, so that a risk averse individual may very likely prefer $D_{2}$ to design $D_{3}$ [Fabrycky and Thuesen, 1980; Pratt, 1964]. Likewise, design $D_{4}$ has a larger expected cost than design $D_{2}$ but a smaller cost variance, so that one might prefer design $D_{4}$ over $D_{2}$.

Table 2 reports the regret $R_{i j}=C_{i j}-L_{i}$ associated with each design choice $D_{j}$ and future demand condition $q_{i}$. Regret is another metric for comparing the cost effectiveness of competing design alternatives. In this particular example,

TABLE 2. Regret of Each Design-Outcome Combination and Design Comparison Based Upon Regret

\begin{tabular}{lrrrrr}
\hline & \multicolumn{5}{c}{ Regret $R_{i j}$ for Design $D_{j}$} \\
\cline { 2 - 5 } & $D_{1}$ & $D_{2}$ & \multicolumn{1}{c}{$D_{3}$} & $D_{4}$ & $L_{i}$ \\
\hline Future demand & & & & & \\
$\quad$ condition, $q_{i}$ & 0 & 30 & 50 & 15 & 60 \\
$\quad q_{1}$ & 25 & 0 & 5 & 20 & 30 \\
$q_{2}$ & 30 & 10 & 0 & 15 & 20 \\
$q_{3}$ & 30 & 10 & 10 & 0 & 25 \\
$q_{4}$ & 40 & 30 & 50 & 20 & \\
Maximum regret* & 26 & 10 & 8 & 13 & \\
Expected regret $\dagger$ & 79 & 60 & 211 & 46 & \\
Variance of regret & & & &
\end{tabular}

*Best design $\mathrm{D}_{4}$.

†Best design $D_{3}$.

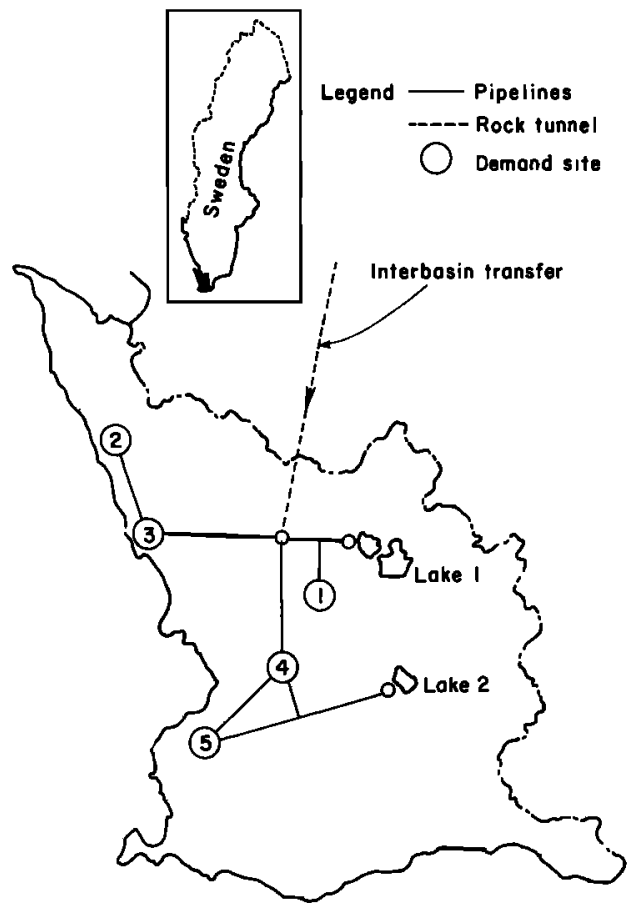

Fig. 2. Diagram of water supply system of southwestem Skane, Sweden, considered in this study.

design $D_{4}$ achieves the minimum maximum regret. Because design $D_{3}$ achieved the minimum expected cost, it also achieves the minimum expected regret [Benjamin and Cornell, 1970, pp. 585-586]. However, one may again want to trade off expected regret with the variance of regret reflecting a desire not to select a design whose performance will be too far from that of the most cost effective design. Hence design $D_{3}$ may be inferior to $D_{2}$, which in turn may be inferior to $D_{4}$.

Minimizing the maximum cost or regret, or minimizing the expected value of either project cost or regret, are all reasonable criteria for project selection. However, each has its drawbacks. The min/max criterion focuses only the worst possible outcome that can result from each design selection, regardless of the probability or likelihood of that event. The expected value criterion looks only at the averge return and ignores risk aversion. When supplemented with a measure of dispersion such as the variance of costs, the approach is improved, but one often does not know how to trade off increases in expected costs for decreased cost variance: design $D_{3}$ versus $D_{2}$ and $D_{2}$ versus $D_{4}$. Even then, as Hashimoto et al. [this issue] show, these two statistics need not be an adequate summary of the entire distribution of possible outcomes.

Table 3 reports the values of the $R_{\beta}$ robustness criterion for several reasonable values of $\beta$. Suppose that one is concerned about design decisions within $\beta=20 \%$ of the cost effective alternative because one's cost estimates have that level of imprecision or because the public and other interested parties will be relatively unconcerned with such modest inefficiencies. Then design $D_{3}$ is very attractive because it has a 70\% probability of achieving that level of cost efficiency. To use a less stringent standard, one could consider future demand conditions that result in opportunity costs in excess of $50 \%$ of the least cost design. Then design $D_{2}$ is 
TABLE 3. Design Selection Based on System Robustness

\begin{tabular}{cccccc}
\hline \multirow{2}{*}{$\begin{array}{c}\text { Robustness } \\
\text { Level, } \beta\end{array}$} & \multicolumn{3}{c}{ Design, $D_{j}$} & $\begin{array}{c}\text { Most Robust } \\
\text { Design at } \\
\text { Level } \beta\end{array}$ \\
\cline { 2 - 6 } & $D_{1}$ & $D_{2}$ & $D_{3}$ & $D_{4}$ & $D_{3}$ \\
$50 \%$ & 0.10 & 0.20 & 0.70 & 0.20 & $D_{2}$ \\
$70 \%$ & 0.10 & 1.00 & 0.90 & 0.30 & $D_{2}$ \\
$100 \%$ & 0.10 & 1.00 & 0.90 & 0.50 & $D_{2}, D_{3}, D_{4}$ \\
$200 \%$ & 0.30 & 1.00 & 1.00 & 1.00 & Indifferent \\
\hline
\end{tabular}

most attractive, for it appears to have a $100 \%$ probability $\left(R_{0.50}=1.00\right)$ of achieving this level of cost efficiency; design $D_{3}$ is a close second with an $R_{0.50}$ value of 0.90 . Use of the $R_{\beta}$ robustness criterion indicates that designs $D_{1}$ and $D_{4}$ are relatively unattractive, even though they achieve the minimum-maximum cost and minimum-maximum regret, respectively.

\section{Application of Robustness Analysis to a SWedish Water Supply System}

The measure of robustness defined above has been applied to a specific regional water supply system planning problem in southwestern Skane, Sweden (Figure 2). In this area a large-scale interbasin water transfer project was proposed to meet projected water demands. Since the projected demands were uncertain, it was not clear just when and to what extent both local source supply capacity and/or the interbasin transfer should be increased or implemented.

At the time that this decision was made (1970), two local lakes were satisfying a major portion of the water demand of five municipalities. In addition, groundwater served each municipality, but substantial expansion of these sources was not possible. To meet increasing demands, lake water withdrawals could be increased and water could be imported through a tunnel, to be built, from a distant lake.

The interbasin water transfer project does not fit well into a stagewise development planning framework because of its indivisibility. Either the tunnel would be built or it would not. In such a situation it is relevant to ask how long the implementation of this major development should be deferred in expectation of obtaining more information about future demand [Hall et al., 1972]. Two results follow immediately from deferment: (1) The present discounted cost of

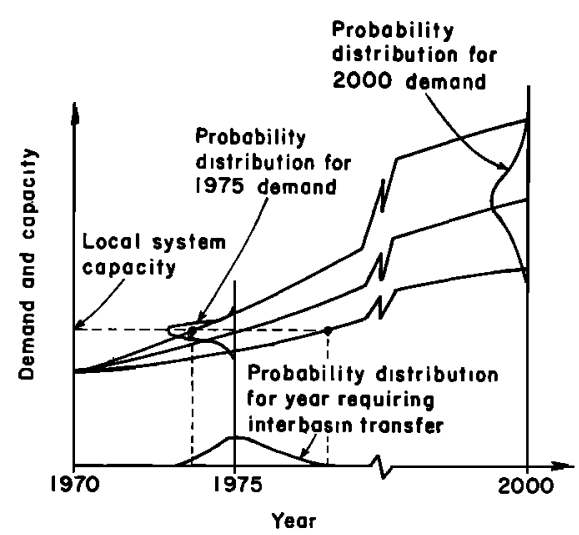

Fig. 3. Drawing shows the 1970 forecast for demand in 1975 and in 2000; uncertainty as to 1975 demand results in a corresponding distribution for the year in which interbasin transfer of water is required. the major development will decrease, first directly from the deferment and second, possibly from reduction in scale of the major development and (2) the cost of the interim development of local supplies will increase because it must provide for the larger demand expected by the time when the major development, i.e., the tunnel, is implemented. Conventional practice is to pick the deferment time that minimizes the total (present discounted) cost of meeting the future demand. The problem is that the demand is uncertain.

Because of the uncertainty in demand, a number of different decisions could be made, each assuming a particular demand projection up to the planning horizon, which was set at the year 2000. Figure 3 illustrates several possible demand trajectories as seen in 1970 and the resulting uncertainty as to when the major development project should be implemented. Of course, possible decisions are not confined to timing or sizing. In the case of low demand, excess water may be diverted to other uses which yield additional benefits, thus reducing the opportunity cost of overdesign. In the case of high demand the demand itself might be reduced using appropriate pricing policies [Kindler et al., 1980].

The range of possible demand trajectories shown in Figure 3 was approximated by seven discrete projections which are characterized by the eventual demand in 1975, denoted

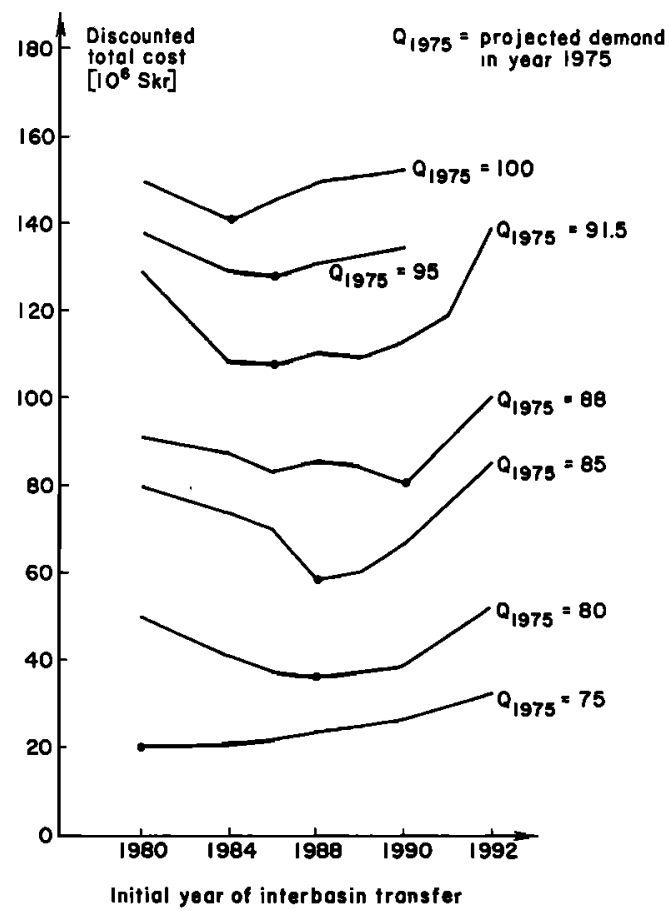

Fig. 4. Discounted total cost of alternative projects as a function of the time of the interbasin transfer under different demand projections for the year 1975 . 
$Q_{1975}$. Each discrete projection has associated with it a minimum cost decision, i.e., the extent of local source development and the timing and size of the tunnel project that are cost effective for that particular demand projection. If indeed a decision is made and the actual future demand is not what was assumed, the discounted total design and operating costs will be higher than expected. The cost functions are shown in Figure 4.

The minimum total costs for the various demand alternative designs define the minimum total cost curve in Figure 5. Also shown in Figure 5 are the cost functions of four alternative development plans, all designed to meet the forecasted demand in year 2000. Design $D_{2}$ is the cost effective plan for the expected value of future demand equivalent to $91.5 \mathrm{Mm}^{3} / \mathrm{yr}$ in 1975 . If indeed the actual demand in year 2000 is as projected in 1970 and hence is equal to $91.5 \mathrm{Mm}^{3} / \mathrm{yr}$ in 1975 , then the design $D_{2}$ will be the cost effective alternative. The total cost function for that alternative is tangent to the minimum cost function at a 1975 demand of $91.5 \mathrm{Mm}^{3} / \mathrm{yr}$ in Figure 5 .

Values of robustness $\boldsymbol{R}_{\beta}$ can be computed for each alternative based on the cost functions shown in Figure 5. Once again, $\boldsymbol{R}_{\boldsymbol{\beta}}$ is the probability that the project costs will be within $100 \beta \%$ of the lowest possible cost of meeting the actual future demand. From Figure 5 one can estimate the $R_{\beta}$ robustness values for the four alternative designs. Table 4 reports $\boldsymbol{R}_{\beta}$ values for three values of $\beta$.

To use the $R_{\beta}$ robustness criterion effectively one must determine the $\beta$ level at which the difference in cost between a particular design and the least cost design is relatively unimportant. It is certainly reasonable to expect that the error in future project construction and operating cost estimates may be $\pm 15 \%$ of the actual costs; this suggests that $\beta$ values of 0.20 or greater may be appropriate. Certainly the
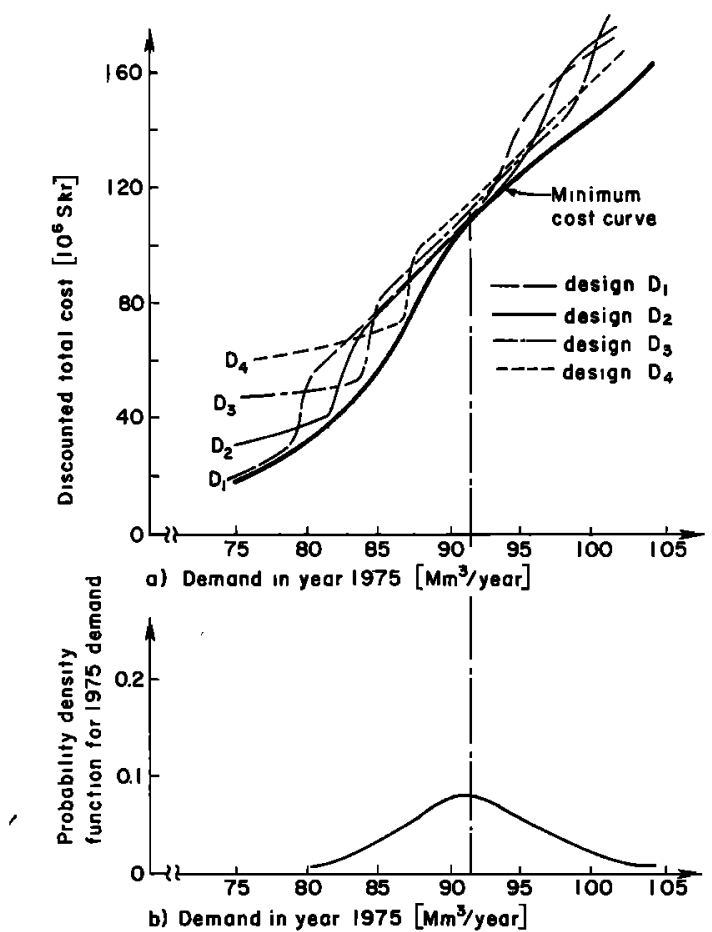

Fig. 5. (a) Discounted total cost functions for minimum-cost and alternative development plans and $(b)$ estimated probability distribution of 1975 future demand.
TABLE 4. Robustness $\boldsymbol{R}_{\beta}$ of Four Alternative Design Implementation Years

\begin{tabular}{ccccc}
\hline & \multicolumn{4}{c}{ Design } \\
\cline { 2 - 5 } $\begin{array}{c}\text { Robustness } \\
\text { Level } \beta\end{array}$ & $D_{1}$ & $D_{2}$ & $D_{3}$ & $D_{4}$ \\
\hline 0.10 & 0.50 & 0.50 & 0.60 & 0.60 \\
0.20 & 0.80 & 0.85 & 0.80 & 0.60 \\
0.50 & 0.95 & 0.98 & 0.90 & 0.80 \\
\hline
\end{tabular}

public and public decision-makers would like to select the most cost effective design for the actual demand conditions that materialize. Unfortunately, this is not always possible given the uncertainty in future demand conditions. This being the case, one can at least discard designs that potentially may perform very poorly. In this instance, $\beta$ defines a cost threshold for poor economic performance.

All of the designs listed in Table 4, except $D_{4}$, have at least an $80 \%$ probability of having their actual costs fall within $\beta=$ $20 \%$ of the estimated minimum possible cost. However, at this $\beta$ value, $D_{2}$ is the most robust design, with an $R_{\beta}$ value of 0.85 . To consider a case where possible system cost performance may be even less satisfactory, line 3 of the table shows that all but design $D_{4}$ have at least a $90 \%$ probability of having their costs fall within $\beta=50 \%$ of the estimated minimum possible costs. Again, at this $\beta$ value, $D_{2}$ is the most robust design with $\boldsymbol{R}_{\mathbf{0 . 5 0}}=\mathbf{9 8 \%}$. Thus with design alternative $D_{2}$ there is only a $2 \%$ probability of relatively very poor cost performance. In this case, both the robustness criterion and cost minimization with the expected 1975 demand point to selection of the same design alternative.

\section{Limitations AND Suggested Further ANALYSIS}

A simple example, but one based on an actual situation, has been presented to illustrate how the robustness measure may be used. This preliminary study has several limitations and suggests the need for further work. In particular:

1. In the above example, only different combinations of three water sources are considered as alternatives. Total cost curves may be more irregular if alternatives with different types of components are compared; for example, surface reservoirs, groundwater, desalinization, or reclaimed wastewater. Naturally, the robustness measures will be more useful in situations where design costs vary more widely among alternatives.

2. Only the uncertainty in future water demand has been integrated into the robustness measure. One could also include in the robustness measure variable energy costs, different interest rates, or more generally, project costs under alternative policies or scenarios [Hashimoto, 1980a].

3. Only physical adjustments (of timing and sizing of the projects) have been considered in the present study. Possible adjustments, however, are not confined to such physical adjustments. Suppose, for instance, that industrial water demand in the region turns out to be lower than originally expected and thus some opportunity cost for overdesign is incurred. This cost might be reduced if the excess water can be diverted to, say, supplementary irrigation which will yield additional benefits. On the other hand, suppose agricultural water demand increases. The cost of making water available to various other uses may increase if no adjustment is made in the face of such an event. Whether such adjustments are possible depends very much on institutional arrangements of 
the region of concern. The flexibility and efficiency of decision-making processes and financing procedures can determine to a certain extent if redesign and reauthorization of the projects are possible in response to changes in the planning environment.

4. One of the most essential tasks for the analysis of robustness is to identify and to describe in appropriate ways those parameters which characterize system inputs. For the water supply system that has been analyzed here, a water demand study should be carefully carried out, taking account of possible changes in future policies. Water demand is as important as water supply when considering the robustness of the entire system. In this respect, the present study is incomplete.

\section{SUMMARY}

In this paper a robustness criterion $R_{\beta}$ is introduced as the probability that the cost of a specific system will be no greater than $1+\beta$ of the cost of the minimum cost design for the realized future demand condition. The difference between the cost of a project and the minimum cost that need be incurred for those particular future demand conditions provides a basis for comparing alternatives. The robustness measure is defined based on this opportunity cost and was applied to planning the expansion of a water supply system under demand uncertainty in southwestern Skane, Sweden.

Acknowledgments. This research was supported in part by a grant from the Office of Water Research and Technology, U.S. Department of the Interior, and by the International Institute for Applied Systems Analysis, Laxenburg, Austria. We gratefully acknowledge the critical comments and suggestions of many, and the contributions of M. Fiering, R. Schuler, and H. Taylor. As always, the opinions expressed are ours and not necessarily those of other individuals or organizations.

\section{REFERENCES}

Benjamin, J. R., and C. A. Cornell, Probability, Statistics and Decision for Civil Engineers, McGraw-Hill, New York, 1970.

Fabrycky, W. J., and J. T. Thuesen, Economic Decision Analysis, Prentice-Hall, Englewood Cliffs, New Jersey, 1980.

Fiering, M. B, The role of systems analysis in water program developments, Natural Res., J., 16, 759-771, October 1976.

Friedman, M., and L. J. Savage, The utility analysis of choices involving risk, J. Pol. Econ., 56, 279-304, 1948.

Hall, W. A., Y. Y. Haimes, and W. S. Butcher, Analysis of feasibility of interim water supplies, Water Resour. Res., 8(2), 317-325, 1972.

Hashimoto, T., Robustness, reliability, resilience and vulnerability criteria for water resources planning, $\mathrm{Ph}$.D. dissertation, Cornell Univ., Ithaca, N. Y., May $1980 a$.

Hashimoto, T., Robustness criterion for planning water supply/ demand systems, Angew. Systemanal. 1(3), $1980 \mathrm{~b}$.

Hashimoto, T., J. R. Stedinger, and D. P. Loucks, Reliability, resiliency and vulnerability criteria for water resource system performance evaluation, Water Resour. Res., this issue.

Loucks, D. P., J. R. Stedinger, and D. A. Haith, Water Resource Systems Planning and Analysis, Prentice-Hall, Englewood Cliffs, New Jersey, 1981.

Matalas, N. C., and M. B Fiering, Water-resource systems planning, in Climate, Climatic Change and Water Supply, National Academy of Sciences, Washington, D. C., 1977.

Pratt, J. W., Risk aversion in the small and in the large, Econometrica, 32(1-2), 122-136, 1964

Raiffa, H., Decision Analysis: Introductory Lectures on Choices Under Uncertainty, Addison-Wesley, Reading, Massachusetts, 1968.

Stigler, G., Production and distribution in the short run, J. Pol. Econ., 47(3), 305-327, 1939.

(Received June 6, 1980;

revised September 4, 1981;

accepted October 2, 1981.) 